Wien klin Mag 2021 $24: 144-151$ https://doi.org/10.1007/s00740-021-00381-5 Online publiziert: 14. Januar 2021 (c) Der/die Autor(en) 2021

\author{
L. H. Lehmann ${ }^{1,2,3} \cdot$ S. Fröhling ${ }^{4,5}$ \\ ' Innere Medizin III, Abteilung für Kardiologie, Pneumologie und Angiologie, Sektion Kardio-Onkologie, \\ Universitätsklinikum Heidelberg, Heidelberg, Deutschland \\ ${ }^{2}$ Deutsches Zentrum für Herz-Kreislauf-Forschung (DZHK), Standort Heidelberg/Mannheim, Heidelberg, \\ Deutschland \\ ${ }^{3}$ Deutsches Krebsforschungszentrum (DKFZ), Heidelberg, Deutschland \\ ${ }^{4}$ Abteilung für Translationale Medizinische Onkologie, Nationales Centrum für Tumorerkrankungen (NCT) \\ Heidelberg und DKFZ, Heidelberg, Deutschland \\ ${ }^{5}$ Deutsches Konsortium für Translationale Krebsforschung (DKTK), Heidelberg, Deutschland
}

\title{
Mechanismen der Kardiotoxizität onkologischer Therapien
}

an ihrer Proliferation zu hindern und/ oder selektiv abzutöten. Hieraus ergibt sich, dass im Prinzip alle Mechanismen, die eine Zelle zum Überleben bzw. zur Teilung benötigt, ein theoretischer Angriffspunkt sind (• Abb. 1). Auf molekularer Ebene haben sich insbesondere die DNA, der Spindelapparat, die Mitochondrien, das Proteasom, bestimmte Kinasen und tumorspezifische Antigene als pharmakologische Ziele etabliert (•Tab. 1).

Eine solche molekulare Einordnung onkologischer Therapien ist sinnvoll, da sich beispielsweise eine Hemmung der DNA-Synthese auf unterschiedliche Art und Weise erreichen lässt, z. B. durch Alkylierung, Interkalation, Einbau von Nukleosidanaloga oder die Hemmung der Purinsynthese oder wichtiger Enzyme wie Topoisomerase II oder Nukleotidreduktase. Alle pharmakologischen Eingriffe folgen einer eigenen Kinetik und Dynamik und haben im Prinzip auch eigene Wirkungen auf Herzmuskelzellen, Gefäße oder kardiale Fibroblasten. Aufgrund der geringen Proliferation der kardialen Zellen ist das Herz relativ gut gegen einen toxischen Schaden geschützt.

\section{Alternative Strategien}

Die Therapie onkologischer Erkrankungen zielt primär darauf ab, maligne Zellen

Dieser Beitrag wurde in der Zeitschrift Der Internist 11·2020 61:1132-1139, https://doi.org/ 10.1007/s00108-020-00881-2 erstpubliziert. Zweitpublikation mit freundlicher Genehmigung der Autoren.
Neben der unspezifischen Hemmung der Proliferation und des Überlebens von Krebszellen haben sich zunehmend andere mögliche Wirkmechanismen einer Tumortherapie entwickelt. So schaffen sich bestimmte Krebsarten eine günstige
Umgebung für ihr Wachstum, z. B. durch Hemmung der lokalen Immunantwort, verstärkte Inflammation oder eine Vermehrung von Gefäßen, die ihrerseits mithilfe gezielter Therapien angegangen werden können (Aktivierung des Immunsystems, Hemmung von Bindegewebszellen und Gefäßwachstum).

Im Umkehrschluss ergeben sich hieraus toxische Wirkungen am Herzen. Außer der Einleitung der Apoptose von Kardiomyozyten kommt es zu Immunphänomenen und Veränderungen der Reizleitung. Phänotypisch führt dies $\mathrm{zu}$ einer reversiblen oder irreversiblen Verschlechterung der linksventrikulären Pumpfunktion (z. B. bei bestimmten Tyrosinkinaseinhibitoren oder Anthrazyklinen), Myokarditiden (z.B. durch Immun-Checkpoint-Inhibitoren) und Arrhythmien (z.B. Vorhofflimmern bei Tyrosinkinaseinhibitoren). Sekundär können aufgrund einer Belastung anderer Organsysteme eine arterielle Hypertonie (z. B. bei Inhibitoren des ,vascular endothelial growth factor", VEGF), eine pulmonalen Hypertonie (z.B. durch Cyclophosphamid) oder kardiale Belastungen im Rahmen von Embolien die Folgen sein.

\section{Anthrazykline}

Die molekulare Wirkung von Anthrazyklinen am Herzen wird v. a. durch direkte Effekte an den Kardiomyozyten erklärt. Drei wesentliche Mechanismen für die 


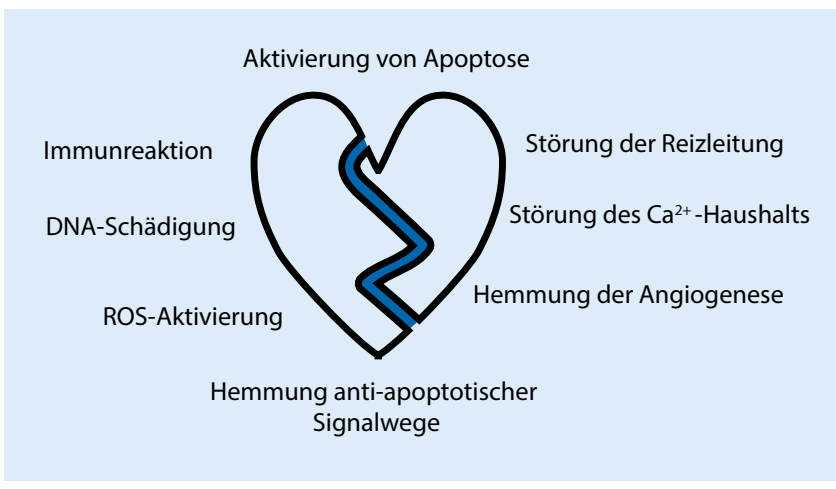

Abb. $1 \varangle$ Häufigste molekulare Mechanismen, die für kardiotoxische Folgen onkologischer Medikamente verantwortlich gemacht werden. ROS "reactive oxygen species" toxische Wirkung werden verantwortlich gemacht:

- Bildung von „reactive oxygen species“ (ROS),

- Aktivierung proapoptotischer Signalwege sowie

- alkylierende Wirkung von Anthrazyklinen auf die Topoisomerase IIb (Top2b) und die DNA selbst (• Abb. 2).

Neue Daten zeigen, dass auch transkriptionellen Prozessen unter Beteiligung von Mikro-RNA und zirkulärer RNA eine Bedeutung bei der Kardiotoxizität zukommt.

\section{》) Die toxische Wirkung der Anthrazykline entfaltet sich in Mitochondrien, im Zytosol und im Zellkern}

Entsprechend den 3 wesentlichen Wirkungsweisen entfaltet sich eine toxische Wirkung in 3 Zellkompartimenten, den Mitochondrien, dem Zytosol und dem Zellkern. Doxorubicin kann an Kardiolipin binden, ein membranständiges mitochondriales Protein, und löst auf diesem Weg eine direkte mitochondriale Schädigung aus. Eine Reduktion der Kardiolipinexpression geht folglich mit einer Protektion von Kardiomyozyten einher [1]. Zudem führt Doxorubicin zur Veränderung der Expression mitochondrialer Gene und zur Generierung intramitochondrialer Superoxide, die letztlich durch verstärkte Mitophagie einen Verlust der Organelle bedingen. Eine Veränderung des mitochondrialen Eisentransports und eine Aktivierung des proa- poptotischen Regulators p53 sind ebenfalls schädigende Effekte. Dem Tumorsuppressor p53 scheint eine bivalente Bedeutung zuzukommen, einerseits als ein Teil des endogenen Reparaturmechanismus, andererseits als Komponente eines überschießenden schädigenden Prozesses bei dauerhafter Inaktivierung. Eine genetische Deletion von p53 kann daher bei niedrig dosierter Gabe von Doxorubicin zu einer Protektion führen, im langfristigen Verlauf aber zu einer dilatativen Kardiomyopathie [2, 3]. Letztlich wird auch die mitochondriale Redoxreaktion direkt gestört, was mit einer Steigerung von ROS einhergeht. Eine direkte Supplementation von Nikotinamidadenindinukleotid $\left(\mathrm{NAD}^{+}\right)$schwächt eine toxische Wirkung ab. Antioxidanzien haben allerdings in Tiermodellen bisher keinen starken kardioprotektiven Effekt gezeigt.

Zytosolische Mechanismen, z.B. ein veränderter $\mathrm{Ca}^{2+}$-Haushalt durch $\mathrm{Re}$ gulation der Expression und Aktivität der $\mathrm{Ca}^{2+}$-Pumpe („sarcoplasmic/ endoplasmic reticulum $\mathrm{Ca} 2+\mathrm{ATPa}$ se“, SERCA2) sowie Dysregulation von Mitochondrien, Autophagie und Fehlregulation von Kinasen/Phosphatasen (z.B. der „ $\mathrm{Ca}^{2+} /$ calmodulin-dependent protein kinase II“, CaMK2) sind ebenfalls an der zytotoxischen Wirkung beteiligt [4]. Die rein zytosolischen Signalwege sind weniger gut untersucht als die nukleären oder mitochondrialen $\mathrm{Me}$ chanismen. Bisher ist zudem unklar, in welcher Beziehung diese wesentlichen 3 molekularen Wirkungsweisen stehen, und ob es klar übergeordnete Mechanismen gibt. Eine Hemmung der ROSGenerierung oder proapoptotischer Proteine oder eine genetische Deletion der Top $2 b$ reduziert in präklinischen Mo- dellen aber die kardiotoxische Wirkung $[5,6]$. Eine klinische Translation dieser Erkenntnisse ist bisher nicht gelungen. Eine neue antiapoptotische Substanz zur Protektion wurde aber kürzlich mit dem klaren Ziel einer zeitnahen klinischen Translation in einem präklinischen Modell parallel zur onkologischen Therapie erfolgreich getestet [5].

Unabhängig von den intrazellulären Mechanismen scheint die Verwendung von liposomalem Doxorubicin ein Eindringen der Anthrazykline in Kardiomyozyten zu reduzieren und damit eine gewisse Kardioprotektion zu ermöglichen [7]. Die Zellmembran und deren Transportmechanismen stellen daher ein zusätzliches Element in der Toxizität von Anthrazyklinen dar.

\section{Proteasominhibitoren}

Neue Proteasominhibitoren, insbesondere Carfilzomib, werden aktuell bei mehreren onkologischen Erkrankungen, insbesondere beim multiplen Myelom, getestet. Im Gegensatz zu den klassischen Inhibitoren (z. B. Bortezomib) hemmt Carfilzomib irreversibel die 20S-Untereinheit des Proteasoms. Diese Inhibition findet auch in Kardiomyozyten statt und ist mutmaßlich an der Entwicklung einer Einschränkung der linksventrikulären Pumpfunktion beteiligt [8]. Die klinische Relevanz dieser Toxizität wird aktuell kontrovers diskutiert; prospektive klinische Daten weisen eher auf eine akzeptable toxische Wirkung hin [9]. Bei Ausweitung der Indikationen wird aber eine abschließende Bewertung der Toxizität abzuwarten sein.

\section{Tyrosinkinaseinhibitoren}

Die Tyrosinkinaseinhibitoren sind eine große Gruppe von Medikamenten, bei der sich je nach molekularem Ansatz typische kardiovaskuläre Komplikationen ergeben. Neben den primären Zielen der Medikamente haben alle häufig verwendeten Tyrosinkinaseinhibitoren (z. B.Dasatinib) mitunter zahlreiche unspezifische Zielstrukturen („off-targets“, [10]).

Dies liegt zum großen Teil an der Hemmung der ATP-Bindungsstelle, die bei vielen Kinasen strukturell ähnlich 
ist und damit die Spezifität der entsprechenden Inhibitoren limitiert. Häufiges Angriffsziel sind die VEGF-Rezeptoren, die mithilfe eines Antikörpers (z.B. Bevacizumab) oder von "small molecules“ (z.B. Sorafenib, Sunitinib und Pazopanib) gehemmt werden, wobei Letztere multiple Kinasen inhibieren. Die Familie der VEGF beinhaltet eine ganze Gruppe von Proteinen (VGEFA, -B, -C, -D, E). Alle Proteine übernehmen eine Funktion bei der Angiogenese durch Kontrolle von Proliferation und Wachstum endothelialer Zellen. Zudem sind VEGF entscheidend für eine regelrechte Herzentwicklung und im adulten Herzen mitverantwortlich für die strukturelle Integrität. Diese wichtige kardiale Funktion wurde bereits frühzeitig im Rahmen genetischer Untersuchungen beschrieben; das onkologische Therapiespektrum wurde erst allmählich erschlossen [11, 12]. Im adulten Herzen ist die Angiogenese auch bei der myokardialen Hypertrophie als kritischer Anpassungsmechanismus notwendig. Eine durch VEGF-Inhibitoren gehemmte Angiogenese dürfte daher bei zusätzlicher kardialer Belastung, z.B. durch arterielle Hypertonie oder vorbestehende Klappenvitien, nachteilig sein.

\section{》) Häufiges Angriffsziel der Tyrosinkinaseinhibitoren sind die VEGF-Rezeptoren}

Außer den Wirkungen am Herzen sind die Effekte auf das Endothel von klinischer Bedeutung, da die Anwendung einer Reihe von Tyrosinkinaseinhibitoren mit der Entwicklung einer arteriellen Hypertonie einhergeht.

Abgesehen von den VEGF-Rezeptoren sind weitere Kinasen etablierte oder potenzielle therapeutische Zielstrukturen in der Onkologie. Hierzu zählen z.B. BCR-ABL1 (Hemmung durch Imatinib und verwandte Substanzen), Bruton-Tyrosinkinase (BTK, Ibrutinib), anaplastische Lymphomkinase (ALK, Crizotinib), „platelet-derived growth factor receptor $A^{\prime \prime}$ (PDGFR $\alpha / \beta$, Imatinib) und „5' AMP-activated protein kinase" (AMPK). Die kardialen Konsequenzen der Inhibition dieser Kinasen

Wien klin Mag 2021 · 24:144-151 https://doi.org/10.1007/s00740-021-00381-5

(c) Der/die Autor(en) 2021

\section{H. Lehmann · S. Fröhling}

\section{Mechanismen der Kardiotoxizität onkologischer Therapien}

\section{Zusammenfassung}

Hintergrund. Onkologische Therapien zeigen am Herz-Kreislauf-System eine Reihe unerwünschter Wirkungen. Insbesondere neuere Therapien sind diesbezüglich unzureichend verstanden, und klinische Daten fehlen bei der Interpretation neuer kardialer Komplikationen.

Ziel der Arbeit. Der vorliegende Beitrag bietet einen Überblick über die Mechanismen kardialer Nebenwirkungen bestimmter onkologischer Therapien.

Material und Methoden. Die Übersichtsarbeit bezieht sich im Wesentlichen auf Daten präklinischer Untersuchungen.

Ergebnisse. Zahlreiche toxische Nebenwirkungen wurde bereits in präklinischen Modellen vorbeschrieben. Für bestimmte Gruppen von Medikamenten (z. B. Anthrazykline, Tyrosinkinaseinhibitoren, Immun-
Checkpoint-Inhibitoren) sind die zugrunde liegenden molekularen Mechanismen aber nicht abschließend geklärt.

Schlussfolgerung. Basierend auf den bekannten molekularen Mechanismen von Kardiotoxizität erschließen sich möglicherweise verbesserte klinische Entscheidungswege. Ein besseres Verständnis ermöglicht auch neue Einblicke in die Pathophysiologie kardialer Erkrankungen. Ziel wird sein, die Ergebnisse translational zu nutzen und in geeigneten kardioonkologischen Einheiten klinisch umzusetzen.

\section{Schlüsselwörter}

Kardioonkologie · Anthrazykline · Doxorubicin - Immun-Checkpoint-Inhibitoren . Herzinsuffizienz

\section{Mechanisms of cardiotoxicity of oncological therapies}

\section{Abstract}

Background. Oncological therapies show a number of undesired adverse effects on the cardiovascular system. In particular, the side effects of recently established oncological therapies are incompletely understood and clinical data are lacking in the interpretation of novel cardiac complications.

Objective. This article provides a short overview of the mechanisms of cardiac side effects of certain oncological therapies. Material and methods. The review is mainly based on data from preclinical studies.

Results. Numerous toxic side effects have already been described and investigated in preclinical models. For certain groups of drugs (e.g. anthracyclines, tyrosine kinase inhibitors and immune checkpoint inhibitors)

the underlying molecular mechanisms are still not fully understood.

Conclusion. An improved understanding of the molecular mechanism involved in cardiotoxicity might help improve the quality of clinical decisions. Additionally, it will provide new insights into the pathophysiology of cardiac diseases. The aim is to use the results of translational research and to clinically implement them in suitable cardio-oncology units.

\section{Keywords}

Cardio-oncology · Anthracyclines · Doxorubicin - Immune checkpoint inhibitor $\cdot$ Heart failure

sind auf molekularer Ebene nicht abschließend geklärt, verschlechtern aber in Tiermodellen im Fall der AMPK und des PDGFR die Stressantwort in Kardiomyozyten. Die Herzen reagieren also verstärkt vulnerabel. Eine Assoziation zu Vorhofflimmern und anderen kardialen Rhythmusstörungen weist des Weiteren auf eine Regulation kritischer Ionenkanäle in Kardiomyozyten hin. Auch bei dieser Substanzgruppe gibt es Hinweise auf eine verstärkte Schädigung kardialer
Mitochondrien und die Generierung von ROS. Klinisch zeigt sich als häufigste Nebenwirkung eine meist transiente Einschränkung der linksventrikulärer Pumpfunktion.

\section{Therapien mit Antikörpern gegen „human epidermal growth factor receptor-2"}

Die alleinige Toxizität von Therapien, die auf Antikörpern gegen „human epider- 
Tab. 1 Onkologische Therapien und wichtigste molekulare Ziele bzw. Mechanismen, die an der Entwicklung unerwünschter kardiovaskulärer Nebenwirkungen beteiligt sind

\begin{tabular}{|c|c|c|c|}
\hline Medikament & Molekulare Mechanismen & Molekulares Ziel & Toxische Wirkung am Herzen \\
\hline Anthrazykline & $\begin{array}{l}\text { Aktivierung von Apoptose; } \mathrm{Fe}^{2+} \text {-Überladung; Störung von Tran- } \\
\text { skription; DNA-Doppelstrangbrüche; Mitophagie; Inflamma- } \\
\text { tion; Autophagie; Energiedepletion }\end{array}$ & Top2b, BAX, ROS & $\begin{array}{l}\text { Apoptose, ROS-Anstieg; kardiale } \\
\text { Dysfunktion; Fibrose }\end{array}$ \\
\hline $\begin{array}{l}\text { Proteasom- } \\
\text { inhibitoren }\end{array}$ & Aktivierung von Apoptose & $\begin{array}{l}\text { 20S-Untereinheit des } \\
\text { Proteasoms }\end{array}$ & $\begin{array}{l}\text { Apoptose, Hypertrophie, Inflam- } \\
\text { mation }\end{array}$ \\
\hline $\begin{array}{l}\text { Immun-Checkpoint } \\
\text { Inhibitoren }\end{array}$ & $\begin{array}{l}\text { Aktivierung des Immunsystems; gestörter } \mathrm{Ca}^{2+} \text {-Haushalt, Apop- } \\
\text { tose? Inflammation }\end{array}$ & PD1, PD-L1, CTLA-4 & Myokarditis; Perikarditis \\
\hline $\begin{array}{l}\text { Tyrosinkinase- } \\
\text { inhibitoren }\end{array}$ & $\begin{array}{l}\text { Hemmung der Zellteilung/Proliferation; endotheliale Dysfunkti- } \\
\text { on; Apoptose; gestörter } \mathrm{Ca}^{2+} \text {-Haushalt; Hemmung der Angioge- } \\
\text { nese }\end{array}$ & $\begin{array}{l}\text { EGFR, VEGF, PDGF, } \\
\text { JAK, RET }\end{array}$ & $\begin{array}{l}\text { Hypertrophie; Kardiomyopathie; } \\
\text { Vorhofflimmern; arterielle Hyperto- } \\
\text { nie }\end{array}$ \\
\hline Immunmodulatoren & $\begin{array}{l}\text { Antiangiogenese, Hemmung von Proliferation, antiinflammato- } \\
\text { risch }\end{array}$ & $\|-6\|-12$, TNF- $a$ & Apoptose; Embolien; Arrhythmien \\
\hline $\begin{array}{l}\text { RAS/RAF/MEK- } \\
\text { Inhibitoren }\end{array}$ & Hemmung der Zellteilung/Proliferation; Apoptose & $\begin{array}{l}\text { Proteine des BRAF- } \\
\text { Signalwegs }\end{array}$ & $\begin{array}{l}\text { Hypertrophie; Fibrose; gestörte } \\
\text { kardiale Entwicklung }\end{array}$ \\
\hline Antikörper & Hemmung der Zellteilung/Proliferation; Apoptose & Oberflächenantigene & Kardiomyopathien \\
\hline CAR-T-Zellen & Einleitung einer gezielten Immunantwort; Inflammation & $\begin{array}{l}\text { Personalisiertes } \\
\text { Antigen }\end{array}$ & Inflammation \\
\hline Zytostatika & $\begin{array}{l}\text { Hemmung der Zellteilung/Proliferation; ROS-Generierung; } \\
\text { Hemmung von Transkription }\end{array}$ & $\begin{array}{l}\text { DNA-Synthese- } \\
\text { Apparat }\end{array}$ & $\begin{array}{l}\text { Kardiomyopathie; Ischämien durch } \\
\text { Vasokonstriktion }\end{array}$ \\
\hline \multicolumn{4}{|c|}{ 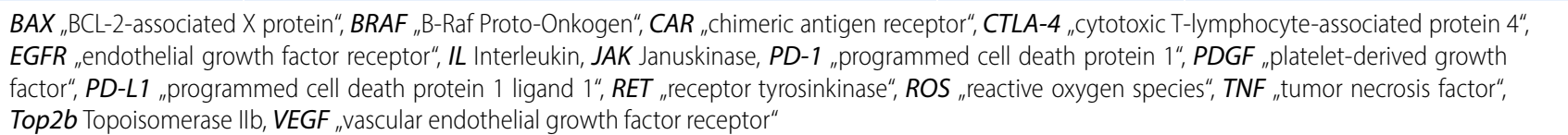 } \\
\hline
\end{tabular}

mal growth factor receptor-2“ (HER2) basieren, ist auf der Basis klinischer Daten nicht eindeutig belegt. Die Therapien werden häufig in Kombination mit oder in der Folge von anthrazyklinbasierten Behandlungen angewendet, sodass die alleinige Toxizität z. B. von Trastuzumab unsicher ist [13]. Aus präklinischen Studien ist bekannt, dass HER2 und seine katalytische Aktivität essenziell für die kardiale Entwicklung sind $[14,15]$. In Herzmuskelzellen des adulten Organismus führt eine dauerhafte genetische HER2-Deletion zum Bild einer dilatativen Kardiomyopathie mit Reduktion der linksventrikulären Pumpfunktion und ausgedünnten Herzwänden [16]. Unterhalb des Rezeptors löst eine Signalkaskade die Aktivierung von MAP-Kinasen aus und ist in essenzielle Funktionen des Kardiomyozyten, wie beispielsweise $\mathrm{Ca}^{2+}$-Handling und mitochondriale Funktion, involviert.

Eine pharmakologische Therapie mit Antikörpern verändert nicht primär die endogene Aktivität des Signalwegs, sondern bewirkt am ehesten die Herabregulation der Rezeptoren. Die reine HER2-Antikörper-Therapie ist daher wahrscheinlich nicht mit einer anhaltenden Reduktion der linksventrikulären Pumpfunktion verbunden. Im Rahmen von präklinischen Studien besteht allerdings der Verdacht auf dauerhafte strukturelle Veränderungen, die möglicherweise eine erhöhte Vulnerabilität im Rahmen nachfolgender Therapien/ Erkrankungen begründen [17].

\section{BRAF- und MEK-Inhibitoren}

Die antiproliferative Wirkung dieser Medikamente beruht auf einer Inhibition des „Mitogen-activated-protein“(MAP)Kinasen-Signalwegs. Mutationen in diesem Signalweg mit der Folge einer unkontrollierten Proliferation sind, z. B. beim malignen Melanom, sehr häufig nachweisbar.

In Kardiomyozyten übernehmen die MAP-Kinasen wichtige biologische Funktionen. An diesem Netzwerk beteiligte Kinasen (z. B. „extracellular signalregulated kinases [ERK]1/2“, „Mitogen-Activated Protein Kinase Kinase“ [MEK] oder „Proto-Onkogen B-Raf“, [BRAF]) sind an kardiomyozytärer Hypertrophie, myokardialer Entwicklung und Apoptose beteiligt [18-21]. Eine pharmakologische Inhibition kann bei Patienten zu einer Einschränkung der linksventrikulären Pumpfunktion führen, die insbesondere bei Therapiebeginn auftritt.

\section{Immun-Checkpoint-Inhibitoren}

Immun-Checkpoint-Inhibitoren wirken durch eine Inhibition des Rezeptorligandensystems von T-Zellen [22]. Hierzu gehören „programmed cell death protein 1“ (PD-1), „programmed cell death protein 1 ligand 1 “ (PD-L1) und „cytotoxic T-lymphocyte-associated protein 4 “ (CTLA-4) als Zielproteine. Die Unterbrechung des inhibitorischen Signals auf T-Zellen mithilfe spezifischer Antikörper resultiert in einer Aktivierung und damit in einer inflammatorischen Antwort auf maligne Zellen. Neben dieser gewollten zytotoxischen Reaktion kann diese „künstliche“ T-Zell-Aktivierung zu einer unerwünschten Immunreaktion am Herzen führen (• Abb. 3). Klinisch tritt sie als Perikarditis oder Myokarditis in Erscheinung. Mechanistisch sind eine direkte Invasion aktivierter T-Zellen ins 


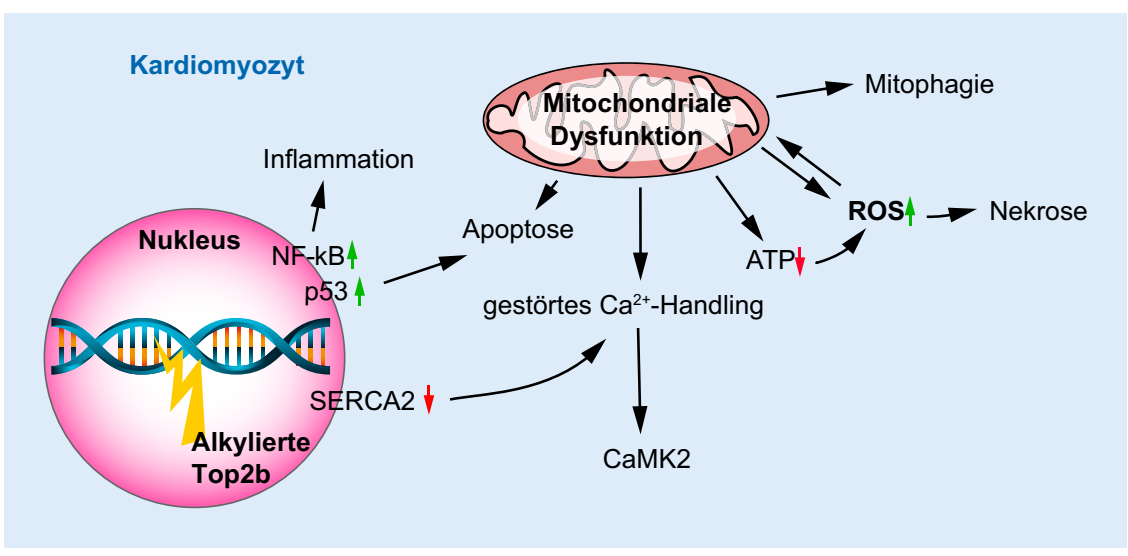

Abb. 2 ム Toxische Wirkungen von Anthrazyklinen am Kardiomyozyten. ATP Adenosintriphosphat, CaMK2 " $\mathrm{Ca}^{2+} /$ calmodulin-dependent protein kinase II", NF- $k B$, nuclear factor ,kappa-light-chain-en-

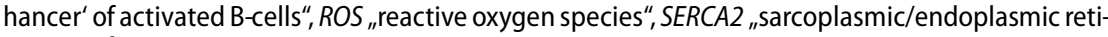
culum $\mathrm{Ca}^{2+}$ ATPase", Top $2 b$ Topoisomerase llb

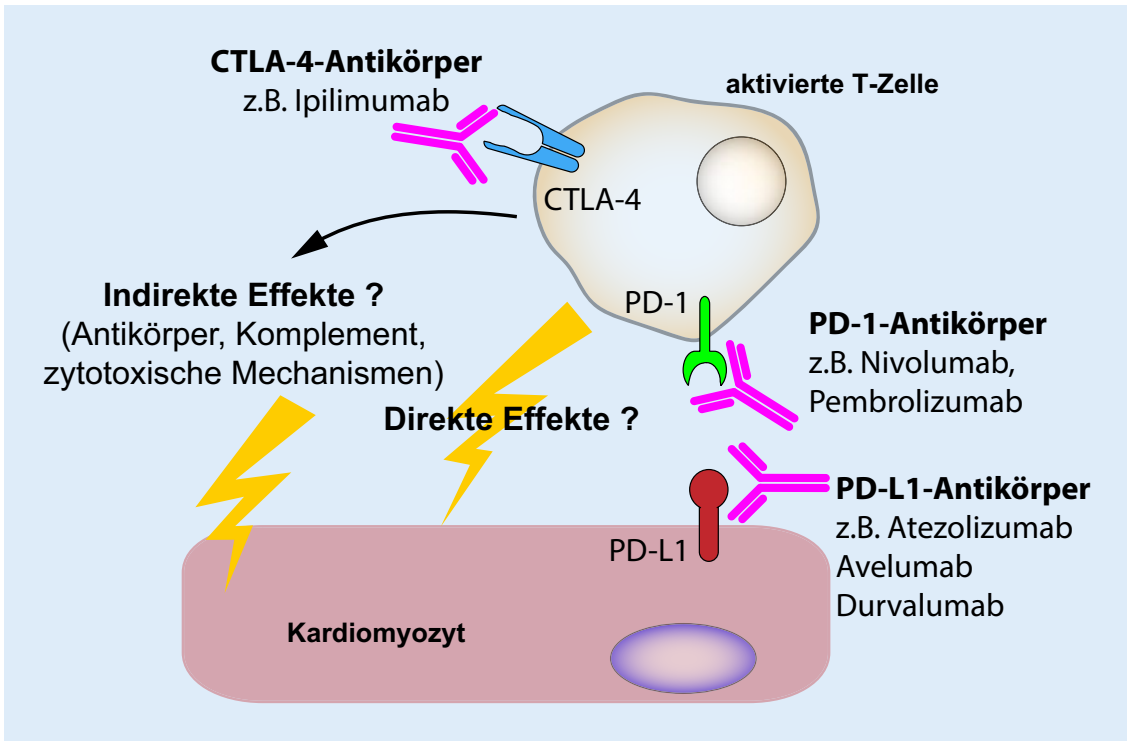

Abb. $3 \Delta$ Toxische Wirkungen von Immun-Checkpoint-Inhibitoren am Kardiomyozyten. CTLA-4 „Cytotoxic T-lymphocyte-associated protein 4", $P D-1$ "programmed cell death protein 1“, $P D-L 1$ „programmed cell death protein 1 ligand $1^{\prime \prime}$

Myokard sowie eine indirekte Immunreaktion mit der Entwicklung von Autoantikörpern gegen kardiales Troponin I beschrieben $[22,23]$.

\section{》) Eine Myokarditis unter Immun-Checkpoint-Inhibitor- Therapie hat eine hohe Mortalität}

Die Erstbeschreibung der globalen genetischen Deletion von PD-1 im Mausmodell durch Nishimura et al. zeigte den Phänotyp einer Myokarditis [24]. Erst im bisher allerdings keine klinische Evidenz. Im Rahmen einer ausgeprägten Myokarditis sind Einzelfallberichte mit einer Reduktion der linksventrikulären Pumpfunktion beschrieben. Diese Patienten sind klinisch besonders schwer betroffen und haben eine deutlich erhöhte Mortalität ( $>50 \%)$. Auch bei einer CD4unabhängigen, globalen genetischen Deletion von CTLA-4 entwickelt sich eine kardiale Inflammation. Basierend auf diesen Daten wird verständlich, dass das Risiko für eine Myokarditis bei Patienten durch eine Kombinationstherapie mit CTLA-4-Antagonisten gemeinsam mit PD-1-/PD-L1-Inhibitoren erhöht ist. Als ein mögliches "Antidot“ einer ungewollten überschießenden Immunreaktion kann ein Antikörper mit CTLA4-Domäne eingesetzt werden (z. B. Abatacept, [26]). Abatacept findet in der Rheumatologie bereits seit vielen Jahren erfolgreich zur Immunmodulation Verwendung. Es hemmt im Fall einer Immun-Checkpoint-assoziierten Myokarditis die künstlich herbeigeführte Aktivierung von T-Zellen.

Unklar sind die prädisponierenden Faktoren für Myokarditis und Perikarditis unter Immun-Checkpoint-Inhibitor Therapie. Eine bereits vorbestehende „Schwäche“ im PD-1, PD-L1- und CTLA-4-System, etwa aufgrund vorbestehender genetischer Mutationen beim Patienten, ist eine Möglichkeit. Bereits vorhandene Antikörper gegen kardiales Troponin werden ebenfalls diskutiert.

Historisch hat sich aus den frühen Mausmodellen (insbesondere PD1-Knockout-Mäuse) die Idee der dilatativen Kardiomyopathie als Immunerkrankung mit der Entwicklung von Autoantikörpern entwickelt [24, 25]. Dies scheint für einen Teil der Patienten mit dilatativer Kardiomyopathie zuzutreffen und ist Gegenstand klinischer und präklinischer Forschung.

\section{Mechanismen der Kardio- toxizität bei Bestrahlung}

Radiotoxizität am Herzen tritt zum einen akut im Rahmen einer inflammatorischen Antwort, zum anderen als chronischer Schaden auf. Die chronischen Schäden führen zur Zunahme 
der Atherosklerose und Häufung von Klappenstenosen. Bindegewebsproliferation und endotheliale Dysfunktion sind wahrscheinlich die Ursachen. Mechanistisch sind die Phänomene bisher nur sehr schlecht untersucht, aber noch am ehesten im Rahmen einer primären endothelialen Schädigung zu verstehen. Es gibt zudem Hinweise auf eine Aktivierung profibrotischer Mechanismen (vermehrte Kollagensynthese, Aktivierung kardialer Fibroblasten u. a.), sodass eine direkte Wirkung von Strahlung auf Fibroblasten ebenfalls wahrscheinlich ist [32].

\section{Ausblick}

Neben den bestehenden präklinischen genetischen Modellen werden v.a. für die Antikörpertherapien zunehmend humanisierte Mausmodelle entwickelt. Hieraus ergeben sich neue Möglichkeiten hinsichtlich der Untersuchung von Kombinationstherapien bzw. kardialen Vorerkrankungen in Kombination mit onkologischen Therapien. Aktuelle klinische Schwerpunkte sind Register zur systematischen Analyse von MultiomicsDaten im Kontext toxischer Nebenwirkungen sowie die Weiterentwicklung der nuklearmedizinischen Bildgebung zur fokussierten Analyse pathologischer Mechanismen (z. B. kardialer Metabolismus, immunologische Reaktionen oder Aktivität von Fibroblasten).

Patienten erhalten meist Kombinationstherapien oder haben bereits potenziell kardiotoxische Therapieregime erhalten. Die dort auftretenden molekularen Mechanismen, bei z. B. latent vorbestehende Schäden an der DNA oder den Mitochondrien, sind häufig therapielimitierend und auf molekularer Ebene kaum verstanden.

\section{Schlussfolgerungen}

Häufig sind potenziell toxische Wirkungen neuer onkologischer Therapien bereits aufgrund präklinischer Modelle zu erwarten. Ein Beispiel ist die Entwicklung der Immun-Checkpoint-Inhibitoren mit dem frühen Nachweis einer Myokarditis im Tiermodell (2001) und der ersten
Dokumentation einer Myokarditis beim Menschen unter Therapie (2017) [24,27].

Ein genaues Verständnis der potenziell toxischen Mechanismen wird zudem die klinische Nachverfolgung und Einschätzung des individuellen Risikos für Patienten beeinflussen, bis es ausreichende klinische Daten gibt [31]. Es ist zudem empfehlenswert die kardiologische Diagnostik in onkologischen Studien frühzeitig auf die zu erwartenden potenziellen toxischen Wirkungen auszurichten, um eine sichere Etablierung neuer Medikamente zu ermöglichen. Für eine Reihe von Therapien in Phase-I-Studien zur Anwendung onkologischer Medikamente wurden bereits kardiale Nebenbefunde in globalen Knockout-Tiere gefunden.

Molekulare Untersuchungen kardiotoxischer Mechanismen werden auch bei „klassischen“ kardialen Pathologien wie beispielsweise der systolischen oder diastolischen Herzinsuffizienz das Verständnis ursächlicher Zusammenhänge ermöglichen.

Neben den Erkenntnissen zu toxischen Wirkungen besteht die Möglichkeit, potenziell kardioprotektive Mechanismen zu verstehen, wie sie beispielsweise bei der Verwendung von Inhibitoren von Histondeacetylasen (HDAC) auf der Basis präklinischer Studien zu vermuten sind [28-30].

\section{Fazit für die Praxis}

- Eine genaue klinische Beobachtung und Dokumentation kardiotoxischer Ereignisse kann entscheidende Impulse für wissenschaftliche Untersuchungen geben.

- Trotz aktueller Empfehlungen zum Umgang mit Patienten mit hohem Risiko zur Entwicklung einer Kardiotoxizität und zum Einsatz spezifischer onkologischer Therapien gibt es für eine hohe Anzahl von Medikamenten nur geringe klinische Erfahrungen bezüglich der Risikostratifizierung und des Monitoring von Patienten.

- Für viele der aktuellen und in Entwicklung befindlichen onkologischen Therapien gibt es aber präklinische Modelle mit möglichen Hinweisen auf Kardiotoxizität. Vor diesem Hintergrund lohnt es sich, die spezifischen molekularen Pathomechanismen zu kennen bzw. weiterzuuntersuchen.

- Zudem ergeben sich aus diesen Mechanismen Hinweise auf den Verlauf von Toxizität und möglichen kardioprotektive Maßnahmen, aus denen auch klinische Fragestellungen für zukünftige kardiologische Studien abgeleitet werden können.

\section{Korrespondenzadresse}

PD Dr. med. L. H. Lehmann

Innere Medizin III, Abteilung für Kardiologie, Pneumologie und Angiologie, Sektion KardioOnkologie, Universitätsklinikum Heidelberg Im Neuenheimer Feld 410, 69120 Heidelberg, Deutschland

Lorenz.Lehmann@med.uni-heidelberg.de

Funding. Open Access funding enabled and organized by Projekt DEAL.

\section{Einhaltung ethischer Richtlinien}

Interessenkonflikt. L.H. Lehmann berät Daiichi Sankyio, Senaca und Servier und erhielt Vortragshonorare von Novartis, Daiichi Sankyio und MSD. S. Fröhling berät Bayer, Illumina, Roche, erhält Forschungsförderung von AstraZeneca, Boehringer Ingelheim, Pfizer, PharmaMar und Roche, sowie Unterstützung von Amgen, Eli Lilly, Illumina, PharmaMar und Roche und erhielt Vortragshonorare von Amgen, Eli Lilly, PharmaMar, sowie Roche.

Für diesen Beitrag wurden von den Autoren keine Studien an Menschen oder Tieren durchgeführt. Für die aufgeführten Studien gelten die jeweils dort angegebenen ethischen Richtlinien.

Open Access. Dieser Artikel wird unter der Creative Commons Namensnennung 4.0 International Lizenz veröffentlicht, welche die Nutzung, Vervielfältigung, Bearbeitung, Verbreitung und Wiedergabe in jeglichem Medium und Format erlaubt, sofern Sie den/die ursprünglichen Autor(en) und die Quelle ordnungsgemäß nennen, einen Link zur Creative Commons Lizenz beifügen und angeben, ob Änderungen vorgenommen wurden.

Die in diesem Artikel enthaltenen Bilder und sonstiges Drittmaterial unterliegen ebenfalls der genannten Creative Commons Lizenz, sofern sich aus der Abbildungslegende nichts anderes ergibt. Sofern das betreffende Material nicht unter der genannten Creative Commons Lizenz steht und die betreffende Handlung nicht nach gesetzlichen Vorschriften erlaubt ist, ist für die oben aufgeführten Weiterverwendungen des Materials die Einwilligung des jeweiligen Rechteinhabers einzuholen.

Weitere Details zur Lizenz entnehmen Sie bitte der Lizenzinformation auf http://creativecommons.org/ licenses/by/4.0/deed.de. 


\section{Literatur}

1. Aryal B, Rao VA (2016) Deficiency in cardiolipin reduces doxorubicin-induced oxidative stress and mitochondrial damage in human B-lymphocytes. PLoSONE 11(7):e158376. https://doi.org/10.1371/ journal.pone. 0158376

2. Li J, Wang PY, Long NA, Zhuang J, Springer DA, Zou J, Lin Y, Bleck CKE, Park JH, Kang JG, Hwang PM (2019) p53 prevents doxorubicin cardiotoxicity independently of its prototypical tumor suppressor activities. Proc Natl Acad SciUSA 116(39):19626-19634. https://doi.org/10.1073/ pnas. 1904979116

3. Zhu W, Zhang W, Shou W, Field LJ (2014) P53 inhibition exacerbates late-stage anthracycline cardiotoxicity. Cardiovasc Res 103(1):81-89. https://doi.org/10.1093/cvr/cvu118

4. Tscheschner $H$, Meinhardt $E$, Schlegel $P$, Jungmann A, Lehmann LH, Muller OJ, Most P, Katus HA, Raake PW (2019) CaMKII activation participates in doxorubicin cardiotoxicity and is attenuated by moderate GRP78 overexpression. PLOS ONE 14(4):e215992. https://doi.org/10.1371/journal. pone. 0215992

5. Amgalan D, Garner TP, Pekson R, Jia XF, Yanamandala $M$, Paulino V, Liang FG, Corbalan JJ, Lee J, Chen $Y$, Karagiannis GS, Sanchez LR, Liang $H$, Narayanagari S-R, Mitchell K, Lopez A, Margulets V Scarlata M, Santulli G, Asnani A, Peterson RT, Hazan RB, Condeelis JS, Oktay MH, Steidl U, Kirshenbaum LA, Gavathiotis E, Kitsis RN (2020) A small-molecule allosteric inhibitor of BAX protects against doxorubicin-induced cardiomyopathy. Nat Cancer 1:315-328. https://doi.org/10.1038/ s43018-020-0039-1

6. Zhang S, Liu X, Bawa-Khalfe T, Lu LS, Lyu YL, Liu LF, Yeh ET (2012) Identification of the molecular basis of doxorubicin-induced cardiotoxicity. Nat Med 18(11):1639-1642. https://doi.org/10.1038/nm. 2919

7. Deng S, Yan T, Jendrny C, Nemecek A, Vincetic M, Godtel-Armbrust U, Wojnowski L (2014) Dexrazoxane may prevent doxorubicin-induced DNA damage via depleting both topoisomerase II isoforms. BMC Cancer 14:842. https://doi.org/10. 1186/1471-2407-14-842

8. Heckmann MB, Doroudgar S, Katus HA, Lehmann LH (2018) Cardiovascular adverse events in multiple myeloma patients. J Thorac Dis 10(35):S4296-S4305. https://doi.org/10.21037/ jtd.2018.09.87

9. Cornell RF, Ky B, Weiss BM, Dahm CN, Gupta DK, Du L, Carver JR, Cohen AD, Engelhardt BG, Garfall AL, Goodman SA, Harrell SL, Kassim AA, Jadhav T, Jagasia M, Moslehi J, O'Quinn R, Savona MR, Slosky D, Smith A, Stadtmauer EA, Vogl DT, Waxman A, Lenihan D (2019) Prospective study of cardiac events during proteasome inhibitor therapy for relapsed multiple myeloma. J Clin Oncol 37(22):1946-1955. https://doi.org/10 1200/JCO.19.00231

10. Karaman MW, Herrgard S, Treiber DK, Gallant $P$ Atteridge CE, Campbell BT, Chan KW, Ciceri $\mathrm{P}$, Davis MI, Edeen PT, Faraoni R, Floyd M, Hunt JP, Lockhart DJ, Milanov ZV, Morrison MJ, Pallares G, Patel HK, Pritchard S, Wodicka LM, Zarrinkar PP (2008) A quantitative analysis of kinase inhibitor selectivity. Nat Biotechnol 26(1):127-132. https:// doi.org/10.1038/nbt1358

11. Carmeliet $P, \mathrm{Ng}$ YS, Nuyens $D$, Theilmeier $G$, Brusselmans K, Cornelissen I, Ehler E, Kakkar VV, Stalmans I, Mattot V, Perriard JC, Dewerchin M, Flameng W, Nagy A, Lupu F, Moons L, Collen D,
D'Amore PA, Shima DT (1999) Impaired myocardial angiogenesis and ischemic cardiomyopathy in mice lacking the vascular endothelial growth factor isoforms VEGF164 and VEGF188. Nat Med 5(5):495-502. https://doi.org/10.1038/8379

12. Haigh JJ (2008) Role of VEGF in organogenesis Organogenesis 4(4):247-256. https://doi.org/10 4161/org.4.4.7415

13. Nemeth BT, Varga ZV, Wu WJ, Pacher P (2017) Trastuzumab cardiotoxicity: from clinical trials to experimental studies. $\mathrm{Br} J$ Pharmacol 174(21):3727-3748. https://doi.org/10.1111/bph. 13643

14. Chan R, Hardy WR, Laing MA, Hardy SE, Muller WJ (2002) The catalytic activity of the ErbB-2 receptor tyrosine kinase is essential for embryonic development. Mol Cell Biol 22(4):1073-1078. https://doi.org/10.1128/mcb.22.4.1073-1078. 2002

15. Lee KF, Simon $\mathrm{H}$, Chen $\mathrm{H}$, Bates $\mathrm{B}$, Hung MC Hauser C (1995) Requirement for neuregulin receptor erbB2 in neural and cardiac development. Nature 378(6555):394-398. https://doi.org/10. 1038/378394a0

16. Ozcelik C, Erdmann B, Pilz B, Wettschureck N Britsch S, Hubner N, Chien KR, Birchmeier C, Garratt AN (2002) Conditional mutation of the ErbB2 (HER2) receptor in cardiomyocytes leads to dilated cardiomyopathy. Proc Natl Acad Sci U S A 99(13):8880-8885. https://doi.org/10.1073/pnas. 122249299

17. ElZarrad MK, Mukhopadhyay $P$, Mohan N, Hao E, Dokmanovic M, Hirsch DS, Shen Y, Pacher P, Wu WJ (2013) Trastuzumab alters the expression of genes essential for cardiac function and induces ultrastructural changes of cardiomyocytes in mice. PLoS One 8(11):e79543. https://doi.org/10.1371/ journal.pone.0079543

18. Bueno OF, De Windt $\amalg$, Tymitz KM, Witt SA Kimball TR, Klevitsky R, Hewett TE, Jones SP, Lefer DJ, Peng CF, Kitsis RN, Molkentin JD (2000) The MEK1-ERK1/2 signaling pathway promotes compensated cardiac hypertrophy in transgenic mice. EMBO J 19(23):6341-6350. https://doi.org/ 10.1093/emboj/19.23.6341

19. Ramirez MT, Sah VP, Zhao XL, Hunter JJ, Chien KR, Brown JH (1997) The MEKK-JNK pathway is stimulated by alpha1-adrenergic receptor and ras activation and is associated with in vitro and in vivo cardiac hypertrophy. J Biol Chem 272(22):14057-14061. https://doi.org/10.1074/ jbc.272.22.14057

20. Wang Y (2007) Mitogen-activated protein kinases in heart development and diseases. Circulation 116(12):1413-1423. https://doi.org/10.1161/ CIRCULATIONAHA.106.679589

21. Harris IS, Zhang S, Treskov I, Kovacs A, Weinheimer C, Muslin AJ (2004) Raf-1 kinase is required for cardiac hypertrophy and cardiomyocyte survival in response to pressure overload. Circulation 110(6):718-723. https://doi.org/10.1161/01.CIR. $0000138190.50127 .6 \mathrm{~A}$

22. Hu JR, Florido R, Lipson EJ, Naidoo J, Ardehali R, Tocchetti CG, Lyon AR, Padera RF, Johnson DB, Moslehi J (2019) Cardiovascular toxicities associated with immune checkpoint inhibitors. Cardiovasc Res 115(5):854-868. https://doi.org/10.1093/cvr/ cvz026

23. Bockstahler M, Fischer A, Goetzke CC, Neumaier HL, Sauter M, Kespohl M, Muller AM, Meckes C, Salbach $C$, Schenk $M$, Heuser A, Landmesser $U_{t}$ Weiner J, Meder B, Lehmann L, Kratzer A, Klingel K, Katus HA, Kaya Z, Beling A (2020) Heartspecific immune responses in an animal model of autoimmunerelated myocarditis mitigated by an immunoproteasome inhibitor and genetic ablation. Circulation. https://doi.org/10.1161/ CIRCULATIONAHA.119.043171

24. Nishimura $H$, Okazaki T, Tanaka $Y$, Nakatani $K$, Hara M, Matsumori A, Sasayama S, Mizoguchi A Hiai H, Minato N, Honjo T (2001) Autoimmune dilated cardiomyopathy in PD-1 receptor-deficient mice. Science 291(5502):319-322.https://doi.org/ 10.1126/science.291.5502.319

25. Okazaki T, Tanaka Y, Nishio R, Mitsuiye T, Mizoguchi A, Wang J, Ishida M, Hiai H, Matsumori A, Minato N, Honjo T (2003) Autoantibodies against cardiac troponin I are responsible for dilated cardiomyopathy in PD-1-deficient mice. Nat Med 9(12):1477-1483. https://doi.org/10.1038/nm955

26. Salem JE, Allenbach Y, Vozy A, Brechot N, Johnson DB, Moslehi JJ, Kerneis M (2019) Abatacept for severe immune checkpoint inhibitor-associated myocarditis. N Engl J Med 380(24):2377-2379. https://doi.org/10.1056/NEJMc1901677

27. Moslehi JJ, Johnson DB, Sosman JA (2017) Myocarditis with immune checkpoint blockade. N Engl J Med 376(3):292. https://doi.org/10.1056/ NEJMc1615251

28. Cao DJ, Wang ZV, Battiprolu PK, Jiang N, Morales CR, Kong Y, Rothermel BA, Gillette TG, Hill JA (2011) Histone deacetylase (HDAC) inhibitors attenuate cardiac hypertrophy by suppressing autophagy. Proc Natl Acad Sci U S A 108(10):4123-4128. https://doi.org/10.1073/pnas.1015081108

29. Berry JM, Cao DJ, Rothermel BA, Hill JA (2008) Histone deacetylase inhibition in the treatment of heart disease. Expert Opin Drug Saf 7(1):53-67. https://doi.org/10.1517/14740338.7.1.53

30. Lehmann LH, Worst BC, Stanmore DA, Backs (2014) Histone deacetylase signaling in cardioprotection. Cell Mol Life Sci 71(9):1673-1690. https:// doi.org/10.1007/s00018-013-1516-9

31. Rassaf T, Totzeck M, Backs J, Bokemeyer C, Hallek M, Hilfiker-Kleiner D, Hochhaus A, Luftner D, Muller OJ, Neudorf U, Pfister R, von Haehling $S$ Lehmann LH, Bauersachs J, Committee for Clinical Cardiovascular Medicine of the German Cardiac Society (2020) Onco-cardiology: consensus paper of the German cardiac society, the German society for pediatric cardiology and congenital heart defects and the German society for hematology and medical oncology. Clin Res Cardiol. https://doi. org/10.1007/s00392-020-01636-7

32. Heckmann MB, Reinhardt F, Finke D, Katus HA Haberkorn U, Leuschner F, Lehmann LH (2020) Relationship between cardiac fibroblast activation protein activity by positron emission tomography and cardiovascular disease. Circ Cardiovasc Imag 13(9) 
Hier steht eine Anzeige.

\section{曾 Springer}

